\title{
Políticas públicas y su contribución en las relaciones de género: el caso de las jornaleras de la Sierra Negra poblana
}

\author{
Public policies and their contribution to \\ gender relationships: A case study on \\ agricultural female workers in Sierra Negra \\ in Puebla
}

\author{
Humberto Piñón-Vargas* \\ Esteban Martínez-Dajui* \\ Miguel SÁNCHEZ-HeRnÁNDEZ* \\ GustaVo Barrientos-Lavín**
}

\begin{abstract}
The objective of the research was to determine the effect of the Secretariat of Social Development-Agricultural Worker's Program, in gender's position and condition of women agricultural laborer families in the Sierra Negra of Puebla. The investigation was descriptive and used a non-probabilistic stratified design. The research techniques included surveys, in-depth interviews, and a study of both weights and measures, and time and movements. The results show that health variable (25\% of the variables related to women's condition) and resources control, decision making, and family violence variables (75\% related to women's position), positively influenced gender equity.
\end{abstract}

Keywords: Sedesol-PAJA, women's practical and strategic needs, agricultural workers, women's projects, Sierra Negra of Puebla.

\section{Resumen}

El objetivo de esta investigación es determinar el efecto del Programa de Atención a Jornaleros Agrícolas de la Secretaría de Desarrollo Social, en la condición y posición de género, de mujeres de la Sierra Negra de Puebla. El estudio fue de tipo descriptivo con diseño probabilístico estratificado. Se realizaron encuestas, entrevistas, estudio de pesas-medidas y de tiempos-movimientos. Los resultados muestran que la variable salud ( $25 \%$ de las variables inherentes a la condición de género), y las de control de recursos, toma de decisiones y violencia familiar ( $75 \%$ de las relativas a la posición de género), influyeron de manera positiva en la equidad de género.

Palabras clave: Sedesol-PAJA, necesidades prácticas y estratégicas de las mujeres, jornaleros(as) agrícolas, proyectos para mujeres, Sierra Negra de Puebla.

* Colegio de Postgraduados, Campus Puebla, México. Correos-e: hpinon@colpos.mx, edajui@ colpos.mx, msanchez@colpos.mx.

** Universidad de las Américas, Puebla, México. Correo-e: gustavoe.barrientos@udlap.mx. 


\section{Introducción}

El presente trabajo tiene la finalidad de investigar los efectos de los proyectos implementados por la Secretaría de Desarrollo Social, a través del Programa de Atención a Jornaleras Agrícolas (Sedesol- PAJA), en las necesidades prácticas o condición y estratégicas o posición de género de las mujeres jornaleras en la Sierra Negra de Puebla (Molyneux, 1985: $227-$ 254; Moser, 1989: 37-54; Young, 1997: 103).

En la región de la Sierra Negra, donde se ubica 17.4\% de los municipios en extrema pobreza del estado de Puebla, se concentra una población preponderantemente indígena nahua de alrededor de 160,200 personas, que constituye a su vez $2.97 \%$ de la población total de la entidad. Más de $50 \%$ de esta población tiene ingresos que la ubican por debajo de la línea de pobreza alimentaria (mapa I) (Conapo, 2006; INEGI, 2006).

Esta situación de marginalidad, asociada a la falta de fuentes de empleo in situ, ha obligado a desarrollar diferentes estrategias de subsistencia, entre las que sobresale el trabajo de jornalero agrícola. Los asalariados rurales presentan como una de sus características el fenómeno migratorio hacia lugares de corte de cultivos agroindustriales. Entre los más recurrentes para la Sierra Negra, están los realizados a las zonas de abastecimiento del ingenio de Calipam en el Valle de Tehuacán, Puebla; los efectuados hacia los ingenios de los municipios de Tezonapa, Omealca y Mahuixtlán, en Veracruz; y al ingenio de Loma Bonita, en Oaxaca. Al mismo tiempo se distinguen otras rutas migratorias a las zonas cafetaleras de la región de Córdoba y Huatusco, Veracruz, y otras migraciones incipientes a estados con agricultura importante en el norte del país, como Sinaloa y Sonora (Pronjag-Puebla, 1997: 187-123).

Otro aspecto que caracteriza el trabajo de los jornaleros agrícolas es el desarrollo de trabajos con elevado desgaste físico, que vinculados con la poca capacidad adquisitiva (producto de salarios paupérrimos y la inseguridad laboral) no sólo se ven reflejados en estados de malnutrición y salud endeble, sino en otros padecimientos inherentes a condiciones de pobreza extrema, como el bajo o nulo nivel escolar, falta de vivienda digna, alto índice de hacinamiento, saneamiento deficiente, capacitación para el trabajo escasa, desconocimiento de derechos fundamentales, entre otros (Pronjag-Puebla, 1997: 187-223).

Sin embargo, las condiciones de vida y trabajo son más precarias entre las mujeres de estas familias de migrantes, ya que desde su posición de desventaja frente a los hombres, tienen que realizar varias y diversas tareas, tanto en sus lugares de origen como de trabajo, que refuerzan su condición de desigualdad y subordinación por partida doble y/o triple, esto es: realizan labores domésticas que les son imputadas de manera tradicional 


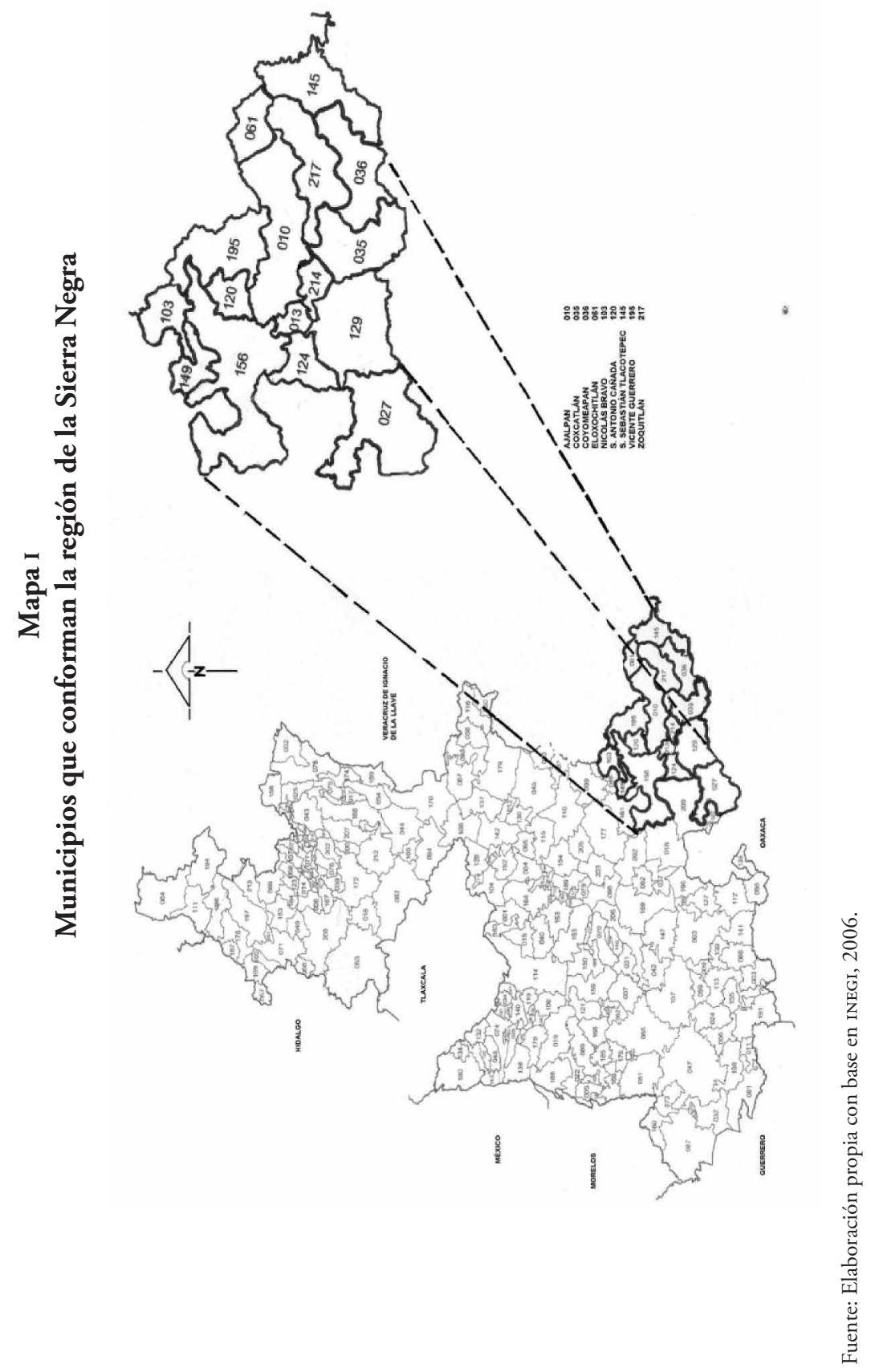


(cocinar, lavar la ropa, atender a los niños, etc.); se incorporan al trabajo productivo laboral como jornaleras, el cual tiene carácter solidario con el jefe de la familia; y en condiciones de escasez como las que viven regularmente, se sacrifican en la distribución de beneficios, como el alimento o cuidados médicos, en favor del resto de los miembros de la familia (Boserup, 1970: 3-19).

El difícil escenario descrito que inscribe a la familia jornalera en procesos de pauperización constante, compromete tanto su reproducción social y biológica como su equidad de género, lo cual se ve potenciado por la instrumentación de inadecuadas políticas públicas, que de manera retórica señalan que intentan reducir desequilibrios en el intercambio social, económico y político entre las personas por razones de sexo, pertenencia étnica o religiosa, bajo el principio de que discrepancias de origen o sociales entre las personas, son injustificables para poder gozar de los derechos universales y aprovechar las oportunidades que se presentan en la vida.

Uno de estos instrumentos de política pública que opera en la Sierra Negra de Puebla desde mediados de 1994, es la Secretaría de Desarrollo Social a través del Programa de Atención a Jornaleros Agrícolas (SedesolPAJA), que establece como uno de sus principios rectores la equidad de género (DOF, 2007: 2; Sedesol, 2007: 13-14).

Por lo anterior, la relevancia de realizar la presente investigación entre las familias jornaleras de la Sierra Negra descansa en diversos motivos, los cuales se exponen a continuación tomando como base algunos criterios formulados por Ackoff (1953) y Miller (1977), adaptados por Hernández et al. (2000:15), a saber: conveniencia, relevancia social y valor teórico.

Con respecto al primer criterio relativo a la conveniencia, se puede señalar que fue significativo el desarrollo de la presente investigación entre las familias asalariadas rurales, en virtud de los propósitos esgrimidos por los propios estudios de género, los cuales no sólo pretenden encontrar explicaciones a las desigualdades existentes entre mujeres y hombres en las diferentes esferas de la vida social y económica, sino que intentan ponerlas de relieve para sensibilizar y concientizar a la sociedad a fin de coadyuvar en la evolución hacia la equidad de género en el contexto de una sociedad dinámica; esto es, que las mujeres y hombres disfruten por igual de los bienes valorados socialmente, las oportunidades, recursos y recompensas.

En segundo lugar, referente a la relevancia social, el trabajo halla su justificación en el ámbito del desarrollo regional, ya que tanto en nuestro país como en el ámbito internacional, el modelo económico predominante, lejos de promover un nivel de vida que permita a los individuos desarrollarse económica, social, cultural y políticamente en un ambiente 
de libertad, con pleno gozo de los derechos individuales, que cumplan con normas y obligaciones que garanticen la convivencia social, ha generado desigualdades importantes por la deficiente distribución de recursos $y$, por ende, graves desequilibrios regionales (Villa, 2008: 15).

En el mismo contexto de relevancia social se puede mencionar que la desigualdad es un problema en sí mismo, que afecta la cohesión social, el crecimiento y el desarrollo económico de un país. En México los desequilibrios se dan en todos los ámbitos (regiones, ciudades, localidades y personas), lo que nos ha colocado entre las 12 primeras sociedades más desiguales del planeta (PR, 2007: 18-22).

Para ilustrar lo anterior se puede mencionar que es evidente la desigualdad tanto al interior de las grandes ciudades como entre las zonas urbanas y rurales, ya que mientras que en la Zona Metropolitana de la Ciudad de México habitan más de 20 millones de personas con los consabidos problemas de contaminación, tráfico e inseguridad, existe una dispersión importante de 184,700 pequeńas localidades rurales de menos de 2,500 habitantes, a las que es difícil dotar de infraestructura y servicios adecuados (INEGi, 2005).

Asimismo, la desigualdad se puede observar con otros indicadores, como la población en condiciones de pobreza alimentaria: mientras que en Chiapas el $47 \%$ se encuentra en este nivel, sólo el 1.3\% se presenta en Baja California (Conapo, 2006; Coneval, 2007).

En ese mismo tenor, los desequilibrios regionales también se hacen presentes al observar los índices de marginación y niveles de pobreza, donde resalta, por ejemplo para el 2005, que de los 103.2 millones de habitantes en el país, $16.4 \%$ habitaba en municipios de alta y muy alta marginación, y del total de municipios existentes $(2,455)$ poco más de la mitad $(50.9 \%)$ se encontraban en estas categorías. De la misma manera, en el estado de Puebla no es menos contrastante la situación, ya que para el año referido, de los 5.3 millones de habitantes en la entidad, $31.3 \%$ se encontraba en niveles de marginalidad alta y muy alta, y $69.1 \%$ del total de sus 217 municipios estaba en esta misma condición.

Dichas condiciones de pobreza y marginalidad generan éxodos migratorios campo-ciudad y campo-campo en busca de mejores formas de vida. Una muestra clara del segundo tipo de migración la encarnan en nuestro país jornaleros agrícolas, quienes son considerados como uno de los sectores más necesitados, y la pobreza que afecta a sus integrantes (mujeres, hombres, niñas y niños) se vive en forma distinta en función de la posición de etnia, edad, sexo y parentesco de los individuos.

Dadas las circunstancias de las mujeres jornaleras en general, asociadas no sólo a su biología (embarazo, lactancia, menstruación, etc.) y a la subordinación culturalmente construida, sino también a sus roles de 
género como cónyuge, madre, ama de casa y especialmente como trabajadora rural, refieren condiciones muy desfavorables que se combinan de manera holística con otras desventajas, producto de las secuelas inherentes a la pobreza misma.

En esta coyuntura, un reto importante es alcanzar un desarrollo que reduzca estos desequilibrios que repercuten en los jornaleros agrícolas, y especialmente en las mujeres jornaleras, para lo cual es importante evocar constructos del PNUD, como su definición del concepto de desarrollo humano: "el proceso de ampliar la gama de opciones de las personas mediante mayor acceso a la educación, atención médica, ingreso y empleo", y el índice de desarrollo humano (IDH) que se construye con base en las dimensiones de longevidad, conocimiento e ingreso per cápita, con lo cual es posible medir el progreso en las condiciones de la humanidad (PNUD, 2003).

Empero, una limitante para el desarrollo humano lo constituye el acceso e igualdad de oportunidades para las mujeres, ya que tradicionalmente han sido discriminadas en cuanto a su participación en el mercado laboral, con menor salario y desigual acceso en la toma de decisiones en las esferas económicas y políticas, pesando además sobre ellas un trabajo invisible y no valorado. La misma Organización de Naciones Unidas en 1996, elaboró el Índice de Desarrollo Relativo al Género (IDG) que refleja desigualdades entre hombres y mujeres en aspectos de salud, educación e ingreso (PNUD, 2004).

$\mathrm{Al}$ respecto, en el año 2002 el IDG nacional fue de 0.7833 , menor al 0.7937 del IDH. En la distribución por entidades federativas, los estados con las menores diferencias entre IDH e IDG o que presentaron la mayor igualdad entre mujeres y hombres fueron el Distrito Federal con 0.8830 y 0.8749 , respectivamente, seguido por Jalisco (0.8007 y 0.7926), Colima (0.8001 y 0.7918$)$, Baja California $(0.8233$ y 0.8147$)$ y Yucatán (0.7778 y 0.7691$)$.

Por otra parte, la mayor diferencia entre IDH e IDG correspondió a Veracruz (0.7457 y 0.7309), seguido por Guerrero (0.7296 y 0.7157$)$, Tabasco $(0.7684$ y 0.7541$)$, Oaxaca $(0.7164$ y 0.7075$)$ y Zacatecas $(0.7563$ y 0.7497 ) (PNUd, 2004).

De manera específica, los resultados para el estado de Puebla (0.7598 de IDH y 0.7497 de IDG) lo colocan en el vigésimo quinto lugar en el ámbito nacional, no muy alejado de las entidades mencionadas en el párrafo precedente. Ahora bien, con respecto a la Sierra Negra, cinco de sus nueve municipios se ubicaron en el rango de muy bajo IDG: Ajalpan ocupó el lugar 187 (de los 217 municipios del estado) con 0.5758, Vicente Guerrero el lugar 209 con 0.5062, Zoquitlán el 211 con 0.4730, Coyomeapan el 213 con 0.4658 y Eloxochitlán el 215 con 0.4237 (PNUD, 2004). 
Dejando de lado la justificación en el término de relevancia social, y para el caso de la justificación en términos de valor teórico, la investigación ofreció la posibilidad de una exploración fructífera en el campo de los estudios de familia; pero especialmente brindó la oportunidad de aportar elementos para enriquecer la teoría de género a partir del estudio de variables poco estudiadas entre uno de los sectores sociales más vulnerables del país: los(as) jornaleros(as) agrícolas.

En otro orden de ideas, se puede mencionar que a partir de las desigualdades de género, se ha propuesto la condición y la posición como categorías analíticas de género. A la condición normalmente se le vincula con intereses y necesidades prácticas, que son resultado de carencias materiales y la falta de satisfacción de necesidades básicas, además de estar orientadas a facilitar el cumplimiento de los roles tradicionales de mujeres y hombres (Carbajal, 1998: 14-15; Martínez et al., 2002: 33-34).

Por su parte, la posición de género estudia el lugar que ocupan las mujeres en relación con los hombres debido a la marginación o negación de los derechos y libertades de una persona por el hecho de ser mujer, independientemente de su estado civil, raza, religión, etnia, etc. En otras palabras, se le asocia a necesidades estratégicas como la ubicación, el reconocimiento social y al estatus asignado a las mujeres en relación con los hombres (Carbajal, 1998: 14-15; Martínez et al., 2002: 33-34).

La investigación reveló que las acciones realizadas por la Sedesol-pajA, tuvieron un efecto positivo en la posición y condición de la mujer jornalera de la Sierra Negra de Puebla, pero no como un objetivo primordial, sino como una consecuencia subyacente de la atención de las necesidades de la familia en general.

\section{Materiales y métodos}

El objetivo del estudio fue conocer el efecto de la implementación de proyectos dirigidos a mujeres de la Sedesol-PAja en las necesidades prácticas y estratégicas de las familias jornaleras de la Sierra Negra, se establecieron la siguiente hipótesis: los proyectos para mujeres implementados por la Sedesol-paja han contribuido a mejorar la condición o necesidades prácticas, así como la posición o necesidades estratégicas de género entre las mujeres jornaleras de la Sierra Negra.

El estudio fue de tipo descriptivo, transversal y no experimental, en el que se consideró como unidad de análisis a las familias jornaleras que fueron beneficiadas con proyectos para mujeres por parte de la SedesolPAja en la región de la Sierra Negra de Puebla. Para el cálculo de la muestra se construyó una base de datos con la inversión del Programa 
desde el inicio de su operación en la región en el año 1994 hasta 2007 (cuadro 1).

Posteriormente, en la base de datos se ubicaron las comunidades donde se invirtieron recursos en proyectos para mujeres jornaleras por parte de dicho programa; asimismo, se procedió a deflactar los montos de inversión de los ejercicios presupuestales, apoyados en el índice nacional de precios al consumidor (INPC) que publica el Banco de México, tomando el año 1994 como base (http://www.banxico.org.mx) (McEachern, 1998: 75-77).

También se verificó qué comunidades de la base de datos construida continuaban siendo atendidas por parte de la Sedesol-paja, conforme al padrón de la Dirección General de Grupos Prioritarios de Oficinas Centrales de dicha secretaría para el ejercicio 2008, y se priorizó de mayor a menor las comunidades, considerando la cantidad de recursos invertidos en proyectos para mujeres. Como criterio de selección se tomó la mayor cantidad de inversión en proyectos para mujeres por comunidad, la cual correspondió a la comunidad de Cuaxuxpa del municipio de Ajalpan, que ocupó el primer lugar en inversión con $10.2 \%$ del total de los recursos (mapa II y foto I).

Derivado de la construcción de la base de datos y los padrones de población jornalera por localidad de la dependencia gubernamental, se determinó que en esta comunidad existían 200 familias jornaleras, de las cuales $20.5 \%$ fueron incluidas en proyectos para mujeres, $24 \%$ no habían

\section{Cuadro 1}

\section{Resumen de la inversión directa de la Sedesol-paja en el ámbito municipal en la Sierra Negra, 1994-2007}

\begin{tabular}{|c|c|c|c|c|c|c|c|}
\hline \multirow{3}{*}{ Municipios } & \multirow{3}{*}{$\begin{array}{c}\text { Número } \\
\text { de } \\
\text { localidades }\end{array}$} & \multirow{3}{*}{$\begin{array}{l}\text { Número } \\
\quad \text { de } \\
\text { proyectos }\end{array}$} & \multicolumn{4}{|c|}{ Inversión } & \multirow{3}{*}{$\begin{array}{l}\text { Número de } \\
\text { familias } \\
\text { beneficiarias }\end{array}$} \\
\hline & & & \multicolumn{2}{|c|}{ Precios corrientes } & \multicolumn{2}{|c|}{ Precios constantes } & \\
\hline & & & (\$) & $\%$ & (\$) & (\%) & \\
\hline Ajalpan & 33 & 151 & $19^{\prime} 159,667$ & 51.50 & $5^{\prime} 480,550$ & 51.52 & 4,748 \\
\hline Coxcatlán & 6 & 34 & $3 ’ 731,053$ & 10.03 & 1’247,718 & 11.73 & 724 \\
\hline Coyomeapan & 7 & 28 & 4’038,596 & 10.86 & 1’082,526 & 10.18 & 449 \\
\hline Eloxochitlán & 18 & 70 & $5^{\prime} 621,344$ & 15.11 & 1'507,349 & 14.17 & 1,141 \\
\hline $\begin{array}{l}\text { Vicente } \\
\text { Guerrero }\end{array}$ & 5 & 24 & 3'792,499 & 10.19 & 1’041,384 & 9.79 & 697 \\
\hline Zoqutilán & 6 & 12 & 860,968 & 2.31 & 278,716 & 2.62 & 203 \\
\hline Total & 75 & 319 & $37^{\prime} 204,127$ & 100.00 & $10^{\prime} 638,242$ & 100.00 & 7,962 \\
\hline
\end{tabular}

Fuente: Elaboración propia de acuerdo con bases de datos de cierre de Programas Operativos Anuales de la Coordinación Estatal Puebla de la Sedesol-paja. 


\section{Mapa II}

\section{Localización de la comunidad de Cuaxuxpa, Ajalpan, Puebla}

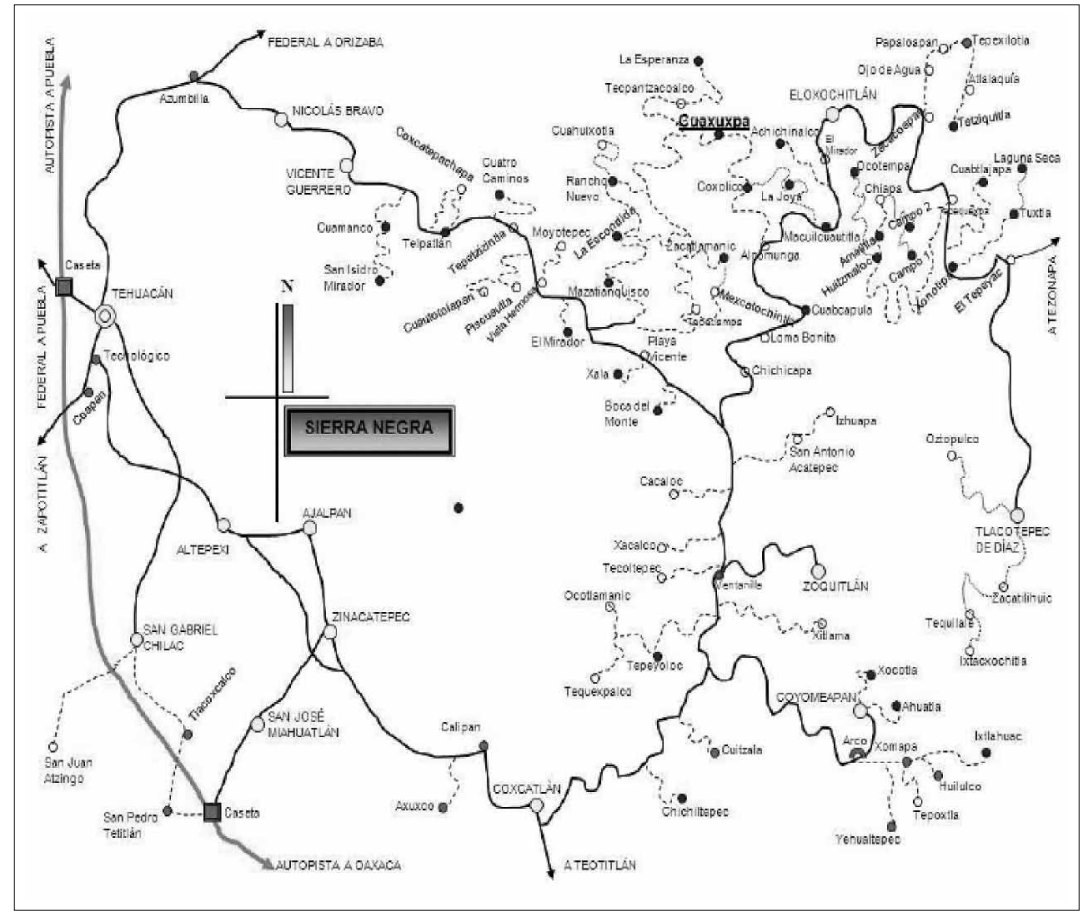

Fuente: Elaboración propia con base en INEGI, 2006.

\section{Foto I}

\section{Comunidad de Cuaxuxpa, Ajalpan, Puebla}

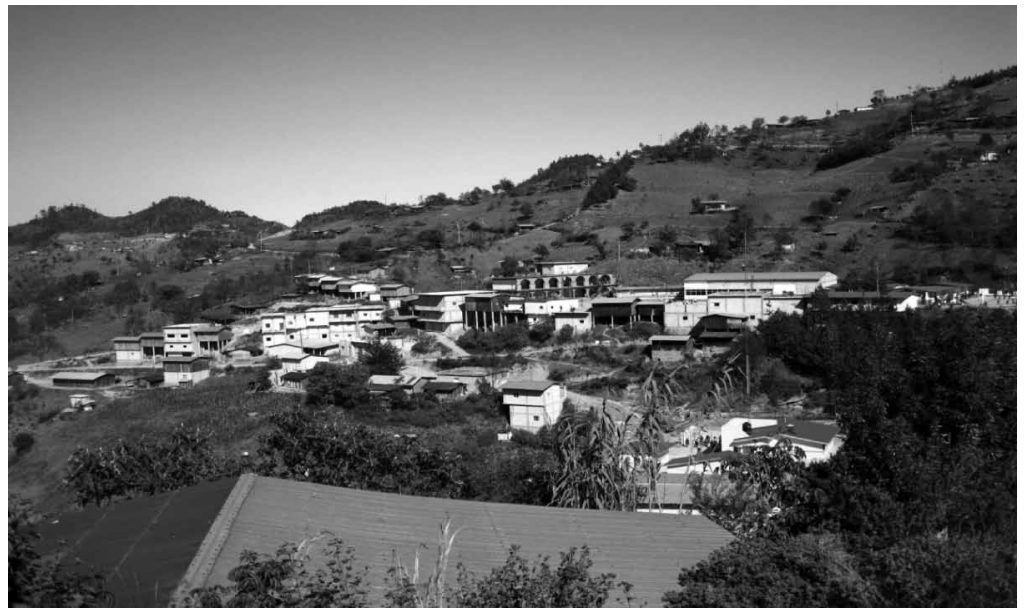

Fuente: Trabajo de campo durante la primavera de 2009. 
sido atendidas con ningún proyecto del Programa y 55.5\% lo fueron mediante otro tipo de proyectos. Por lo anterior, el diseño muestral utilizado fue el probabilístico estratificado, con un nivel de confianza de 95\% y un error estándar de 5 por ciento.

Para determinar el número de muestras por estrato se empleó el método de Kish (Hernández et al., 2000: 209-213), y se decidió trabajar con 59 familias jornaleras en total: el estrato 1 quedó conformado por 12 familias atendidas con proyectos para mujeres por parte de la SedesolPAJA; el estrato 2 por 33 familias incluidas en otro tipo de proyectos de la Sedesol-paja, y el estrato 3 por 12 sin proyectos o no atendidas por la instancia gubernamental (cuadro 2).

Por otra parte, la metodología contempló la elaboración y aplicación de cuatro diferentes tipos de instrumentos exploratorios (encuestas, entrevistas a profundidad, estudio de pesas y medidas, así como estudio de tiempos y movimientos), además de las observaciones realizadas por el investigador que fueron plasmadas en el diario de campo. Las herramientas utilizadas permitieron en su conjunto recopilar información cuantitativa y cualitativa en los renglones establecidos para las variables de posición (división del trabajo, control de recursos, acceso al poder y violencia familiar) y condición (estado nutricional, salud, educación y carencias materiales) (cuadro 3).

La encuesta se aplicó al 100\% de las mujeres jornaleras jefas de familia de los tres estratos; por su parte, la entrevista a profundidad se aplicó a 3\% de las mujeres (dos por estrato) consideradas como clave, lo que permitió obtener datos que apuntalaron la información obtenida mediante la encuesta. Con estas dos herramientas se recogió la información del $100 \%$ de las variables de posición (11 indicadores de las cuatro variables),

\section{Cuadro 2 \\ Estratos y cálculo de la muestra}

\begin{tabular}{|c|c|c|c|c|c|c|c|}
\hline \multirow{2}{*}{ Estrato } & \multirow{2}{*}{$\begin{array}{l}\text { Descripción } \\
\text { del estrato }\end{array}$} & \multirow{2}{*}{$\begin{array}{c}\text { Número } \\
\text { de } \\
\text { familias }\end{array}$} & \multicolumn{5}{|c|}{ Muestreo* } \\
\hline & & & Indice & $\begin{array}{l}\text { Total de } \\
\text { familias }\end{array}$ & Redondeo & $\begin{array}{l}(+-) \\
10 \%\end{array}$ & $\%$ \\
\hline Estrato 1 (E1) & $\begin{array}{l}\text { Con proyectos } \\
\text { para mujeres }\end{array}$ & 41 & 0.2675 & 10.9675 & 11 & 12 & 20.3 \\
\hline Estrato 2 (E2) & $\begin{array}{l}\text { Con otro tipo de } \\
\text { proyectos }\end{array}$ & 111 & 0.2675 & 29.6925 & 30 & 33 & 55.5 \\
\hline Estrato 3 (E3) & $\begin{array}{l}\text { Sin proyecto o no } \\
\text { atendidas }\end{array}$ & 48 & 0.2675 & 12.84 & 13 & 14 & 24.2 \\
\hline Total & 3 & 200 & & 53.5 & 54 & 59 & 100.0 \\
\hline
\end{tabular}

* Nivel de confianza de $95 \%$ y error estándar de 5 por ciento.

Fuente: Elaboración propia. 
y la información de otros 11 indicadores de tres de las cuatro variables de condición (salud, educación y carencias materiales).

Los estudios de pesas-medidas y de tiempos-movimientos que se realizaron para determinar las diferencias en el estado nutricional (encuadradas en las necesidades de condición), proporcionaron información suplemen-

\section{Cuadro 3}

\section{Variables, indicadores e instrumentos aplicados}

\begin{tabular}{|c|c|c|c|}
\hline Dimensiones & Variable & Indicador & Instrumento aplicado \\
\hline \multirow[t]{7}{*}{$\begin{array}{l}\text { Necesidades } \\
\text { estratégicas }\end{array}$} & \multirow[t]{2}{*}{$\begin{array}{l}\text { División del } \\
\text { trabajo }\end{array}$} & $\begin{array}{l}\text { Tipo de actividad desarrollada } \\
\text { por sexo }\end{array}$ & $\begin{array}{l}\text { Encuesta y entrevista a } \\
\text { profundidad }\end{array}$ \\
\hline & & $\begin{array}{l}\text { Carga de trabajo por sexo } \\
\text { Ingreso y distribución del gasto }\end{array}$ & $\begin{array}{l}\text { Estudio de tiempos y } \\
\text { movimientos }\end{array}$ \\
\hline & \multirow{2}{*}{$\begin{array}{l}\text { Control de } \\
\text { recursos }\end{array}$} & Administración del gasto & \multirow{2}{*}{$\begin{array}{l}\text { Encuesta y entrevista } \\
\text { a profundidad }\end{array}$} \\
\hline & & $\begin{array}{l}\text { Acceso a instrumentos } \\
\text { modernos y otros bienes }\end{array}$ & \\
\hline & \multirow{2}{*}{$\begin{array}{l}\text { Acceso al } \\
\text { poder }\end{array}$} & Decisión sobre recursos & \multirow{2}{*}{$\begin{array}{l}\text { Encuesta y entrevista } \\
\text { a profundidad }\end{array}$} \\
\hline & & $\begin{array}{l}\text { Participación en la vida pública } \\
\text { y gestión de apoyos } \\
\text { institucionales }\end{array}$ & \\
\hline & $\begin{array}{l}\text { Violencia } \\
\text { familiar }\end{array}$ & $\begin{array}{l}\text { Padecimiento de prohibiciones, } \\
\text { amenazas, actitudes } \\
\text { devaluatorias, control y } \\
\text { vigilancia por celos, } \\
\text { intimidaciones, y maltrato } \\
\text { verbal, físico y sexual }\end{array}$ & $\begin{array}{l}\text { Encuesta y entrevista } \\
\text { a profundidad }\end{array}$ \\
\hline \multirow{10}{*}{$\begin{array}{l}\text { Necesidades } \\
\text { prácticas }\end{array}$} & Estado & Ingesta de alimento & \multirow{2}{*}{$\begin{array}{l}\text { Estudios de pesas y } \\
\text { medidas, y de tiempos } \\
\text { y movimientos }\end{array}$} \\
\hline & nutricional & Gasto energético & \\
\hline & \multirow[t]{2}{*}{ Salud } & Morbilidad por individuo & \multirow{2}{*}{$\begin{array}{l}\text { Encuesta y entrevista } \\
\text { a profundidad }\end{array}$} \\
\hline & & $\begin{array}{l}\text { Tipo de atención por } \\
\text { enfermedades }\end{array}$ & \\
\hline & \multirow[t]{3}{*}{ Educación } & Analfabetismo & \multirow{3}{*}{$\begin{array}{l}\text { Encuesta y entrevista } \\
\text { a profundidad }\end{array}$} \\
\hline & & Nivel de escolaridad & \\
\hline & & Lengua & \\
\hline & \multirow{3}{*}{$\begin{array}{l}\text { Carencias } \\
\text { materiales }\end{array}$} & Condiciones de vivienda & \multirow{3}{*}{$\begin{array}{l}\text { Encuesta y entrevista } \\
\text { a profundidad }\end{array}$} \\
\hline & & $\begin{array}{l}\text { Servicio sanitario y drenaje, } \\
\text { agua y electricidad }\end{array}$ & \\
\hline & & $\begin{array}{l}\text { Tipo de acceso o camino a la } \\
\text { vivienda }\end{array}$ & \\
\hline
\end{tabular}

Fuente: Elaboración propia. 
taria entre ellos. El primero estuvo encaminado a conocer las diferencias en la ingesta de alimento por parte de hombres y mujeres integrantes de las familias jornaleras, para lo cual se hizo durante tres días seguidos el pesaje de los alimentos ingeridos por cada miembro de la familia a través de una báscula de precisión. Los datos obtenidos se analizaron por medio de tablas de valor nutritivo de alimentos de la Organización Mundial para la Agricultura y la Alimentación (FAO) (Daltabuit, 1988: 69-71). El segundo estudio se realizó con miras a determinar el gasto energético, para lo cual se llevó a cabo el registro del tipo de actividad y tiempo empleado en cada una de las tareas desarrolladas por ambos jefes de familia exclusivamente. Este trabajo se realizó durante las 24 horas de tres días seguidos, con las mismas familias con quienes se realizó el trabajo de ingesta de alimentos (Daltabuit et al., 1988: 84-90).

La herramienta de registro del tiempo y tipo de actividad empleado por hombres y mujeres se basó en el trabajo de Viteri (1971: 418-430), que clasifica las principales actividades de grupos indígenas en: básicas (aseo personal, consumo de alimentos y dormir); productivas (labores culturales, acarreo de leña, cuidado de animales y cuidado de huerto de traspatio); y no productivas (descansar, platicar y jugar) (cuadro 3). El cálculo del consumo energético a partir de la información de campo se realizó con base en tablas de la FAO en coordinación con la Organización Mundial de la Salud (oMs), para países latinoamericanos (FAO-OMS, 1985).

Finalmente, para la interpretación de la medición de todas las variables se utilizó el análisis de frecuencias relativas o porcentajes, y nos apoyamos sobre todo en histogramas para su mejor percepción. Asimismo, debido a la importante cantidad y diversidad de indicadores de las diferentes variables de condición y posición, se optó por traducir al mismo idioma (el matemático) los resultados de las mismas, estandarizando los resultados y construyendo un índice ponderado de género (IPG) para el estudio basado en el elaborado por el PNUD, que por su flexibilidad y relativa independencia correlativa a cada una de las variables, permite castigar o recompensar el efecto de los proyectos para mujeres del Programa en las relaciones de género de las familias estudiadas.

Se destaca que el IPG para la presente investigación se construyó a partir de los subíndices ponderados de condición (SPC) y el subíndice ponderado de posición (SPP); asimismo se enfatiza que el IPG otorga a cada una de las variables el mismo valor, aun cuando existe importante variación en la cantidad de indicadores para construir cada una. En otros términos, considera que cada una de las ocho variables tienen el mismo nivel de injerencia en las relaciones de género entre las familias jornaleras, por lo que no se establece ponderación o valoración arbitraria alguna. 


$$
\begin{aligned}
\mathrm{IPG} & =\frac{\sum((0.5 \text { (variables de condición })+0.5(\text { variables de posición }))}{100} \\
\mathrm{SPC} & =\frac{0.5(\text { alimentación }+ \text { educación }+ \text { salud }+ \text { carencias materiales })}{100} \\
\mathrm{SPP} & =\frac{0.5(\text { div. trabajo }+ \text { control rec. }+ \text { acceso poder }+ \text { viol. fam. })}{100}
\end{aligned}
$$

\section{Resultados y discusión}

Los resultados obtenidos en la investigación de campo a partir de la aplicación de las herramientas, se organizaron en matrices de doble entrada, donde se puede apreciar en forma sencilla la valoración obtenida por cada variable operacionalizada en una escala del uno al tres; lo anterior, de acuerdo con el mayor o menor efecto en la condición o posición de las mujeres jornaleras de Cuaxuxpa, Ajalpan, Puebla.

\subsection{Necesidades prácticas}

Este apartado incluyó la medición u operacionalización de cuatro variables: alimentación, educación, salud y carencias materiales. La primera se determinó a partir de cuatro indicadores, y se encontró que el E2 fue el mejor posicionado, seguido del E3 y por último el E1, lo que indica nula injerencia de la Sedesol-paja en la condición de género. La segunda variable, referente a educación, se determinó a partir de tres indicadores y se halló que las jornaleras del E1 fueron las mejor posicionadas, seguidas de las del E2 y finalmente las del E3 (cuadro 4).

La variable de salud se midió sólo con dos indicadores, donde se encontró que el E1 y el E2 compartieron la mejor posición en cuanto a puntuación, por lo que se considera que no existe evidencia sustancial de un efecto positivo de los proyectos para mujeres de la Sedesol-paja en la condición de género de las familias jornaleras. La cuarta variable, carencias materiales, fue la más amplia de todas, ya que contempló seis indicadores; al respecto, el E1 donde se esperaban mayores efectos en la equidad ocupó el segundo lugar en relación con los otros estratos, por lo que no se considera como positivo el efecto de la política pública en la condición de género (cuadro 5).

Los resultados expuestos en este apartado se pueden interpretar a la luz de las elaboraciones de Moser (1989: 37-54), que apuntan a que el mejoramiento de las necesidades prácticas de las mujeres van más en el sentido de incrementar sus capacidades en el contexto del desarrollo humano que en la equidad de género, ya que responden a un interés inmediato de las mujeres por cumplir con los roles que les son asignados 
Cuadro 4

Resultados de las variables de alimentación y educación por estrato

\begin{tabular}{lllccc}
\hline \multirow{2}{*}{ Variable } & \multicolumn{2}{c}{$\begin{array}{c}\text { Variable } \\
\text { operacionalizada }\end{array}$} & \multicolumn{1}{c}{ Indicador } & \multicolumn{2}{c}{ Lugar por estrato } \\
\cline { 4 - 6 } & Ingesta & Consumo de calorías & 1 & 3 & 2 \\
& & Consumo de proteínas & 1 & 2 & 3 \\
\multirow{3}{*}{ Alimentación } & Gasto & Gasto de calorías & 1 & 3 & 2 \\
& Consumo $v$ s. & Balance & 1 & 3 & 2 \\
& gasto energético & & & & \\
\multirow{3}{*}{ Subtotal } & & & 4 & 11 & 9 \\
\hline \multirow{3}{*}{ Educación } & Lectoescritura & Porcentaje de lectoescritura & 3 & 2 & 1 \\
& Escolaridad & Nivel de escolaridad en años & 3 & 2 & 1 \\
& Lengua & Porcentaje de comprensión del & 3 & 2 & 1 \\
Subtotal & & español & 9 & 6 & 3 \\
\hline
\end{tabular}

Fuente: Elaboración propia con base en datos de investigación de campo durante la primavera de 2009.

por la división de género del trabajo, que comúnmente se asocia a las funciones de madre, esposa y responsable del bienestar familiar, y no entrańan cambios radicales, como la emancipación de las mujeres o empoderamiento.

En otras palabras, no hay avance en el mejoramiento de las condiciones materiales de las mujeres jornaleras y sus familias ubicadas en el E1 por el efecto de los proyectos para mujeres de la Sedesol-paja. Esto lo respaldan los resultados positivos obtenidos únicamente en una variable (educación) de las cuatro medidas. De hecho, la evidencia recogida en campo derivada de la observación directa, apunta a que se deterioró la posición respecto a los hombres, por la percepción de una pérdida de estatus familiar e invisibilización del aporte económico, como consecuencia de su trabajo con los proyectos para mujeres de la Sedesol-paja.

\subsection{Necesidades estratégicas}

Este segundo apartado incluyó la medición de otras cuatro variables, a saber: división del trabajo, control de recursos, acceso al poder y violencia familiar.

La división del trabajo se midió con tres variables operacionalizadas y siete distintos indicadores. Al final del conteo de frecuencias, interpretación y ponderación de cada uno de los indicadores, se encontró que los valores de los tres estratos son idénticos, por lo que no existe efecto im- 


\section{Cuadro 5}

\section{Resultados de las variables de salud y carencias materiales por estrato}

\begin{tabular}{|c|c|c|c|c|c|}
\hline \multirow[t]{2}{*}{ Variable } & \multirow{2}{*}{$\begin{array}{c}\text { Variable } \\
\text { operacionalizada }\end{array}$} & \multirow[t]{2}{*}{ Indicador } & \multicolumn{3}{|c|}{$\begin{array}{l}\text { Lugar por } \\
\text { estrato }\end{array}$} \\
\hline & & & E1 & $E 2$ & E3 \\
\hline \multirow{2}{*}{ Salud } & $\begin{array}{l}\text { Morbilidad } \\
\text { Enfermedades }\end{array}$ & $\%$ incidencia enfermedades & 2 & 3 & 1 \\
\hline & $\begin{array}{l}\text { Atención } \\
\text { enfermedades }\end{array}$ & $\begin{array}{l}\% \text { recursos económicos para la } \\
\text { atención }\end{array}$ & 3 & 2 & 1 \\
\hline \multirow[t]{8}{*}{ Subtotal } & & & 5 & 5 & 2 \\
\hline & Vivienda & Núm. de cuartos/vivienda & 3 & 2 & 1 \\
\hline & & Índice de hacinamiento & 3 & 2 & 1 \\
\hline & & Superficie $\left(\mathrm{m}^{2}\right)$ & 3 & 2 & 1 \\
\hline & & Material de construcción en techos & 3 & 2 & 1 \\
\hline & & $\begin{array}{l}\text { Material de construcción en } \\
\text { paredes }\end{array}$ & 1 & 3 & 2 \\
\hline & & $\begin{array}{l}\text { Material de construcción en pisos } \\
\text { (cocina) }\end{array}$ & 3 & 3 & 3 \\
\hline & & $\begin{array}{l}\text { Material de construcción en pisos } \\
\text { (sala-comedor) }\end{array}$ & 2 & 3 & 1 \\
\hline \multirow[t]{8}{*}{$\begin{array}{l}\text { Carencias } \\
\text { materiales }\end{array}$} & & $\begin{array}{l}\text { Material de construcción en pisos } \\
\text { (cuartos) }\end{array}$ & 1 & 3 & 2 \\
\hline & & Estado de la vivienda & 3 & 2 & 1 \\
\hline & Servicio & Tipo de servicio & 3 & 2 & 1 \\
\hline & Santatio & Condiciones & 3 & 2 & 1 \\
\hline & Servicio agua & Tipo de acceso & 3 & 2 & 1 \\
\hline & Servicio energía & En casa & 1 & 3 & 2 \\
\hline & & Alumbrado público & 2 & 3 & 1 \\
\hline & $\begin{array}{l}\text { Acceso a la } \\
\text { vivienda }\end{array}$ & $\begin{array}{l}\text { Tipo de camino y estado del } \\
\text { mismo }\end{array}$ & 1 & 2 & 3 \\
\hline Subtotal & & & 35 & 36 & 22 \\
\hline
\end{tabular}

Fuente: Elaboración propia con base en datos de investigación de campo durante la primavera de 2009. 
portante en las necesidades estratégicas de las mujeres jornaleras por parte de los proyectos de la Sedesol-paja.

La variable de control de recursos se midió a través de tres distintas variables operacionalizadas y sus respectivos indicadores. Se halló que el E1 obtuvo la más alta recompensa, seguida por el E3 y el E2, lo que permite sostener que hay efecto positivo de la política pública en la posición de género de las jornaleras de Cuaxuxpa (cuadro 6).

Por lo que respecta al acceso al poder, el cual se midió con una variable operacionalizada y tres indicadores, se encontró que el E1 obtuvo la más alta recompensa con base en el análisis e interpretación efectuados. En consecuencia, se considera que hay un efecto positivo del Programa en este aspecto de la posición de las mujeres jornaleras de la comunidad estudiada.

Finalmente, la violencia familiar se determinó con una variable operacionalizada y ocho indicadores, que señalaron que a pesar de la leve diferencia entre la ponderación del E1 con los otros dos estratos, se obtuvo la más alta recompensa. Esto significa un efecto positivo en la posición de las mujeres en los proyectos de la Sedesol-paja (cuadro 7).

Es posible interpretar los resultados de las variables ubicadas en este apartado a partir de lo que señala Young (1997: 105-107), en el sentido de que las necesidades estratégicas se generan del análisis de las relaciones de dominio-subordinación entre los géneros y se refieren a la ubicación social y económica de las mujeres en relación con los hombres, lo que de manera pragmática representa desventajas y menores oportunidades que afectan a las mujeres, derivadas de concepciones culturales que impiden la participación plena e igualitaria en la sociedad.

Así, estos aspectos vinculados a la subordinación de género, que por lo regular requieren intervención externa (para generar procesos participativos, debido a que históricamente se ha demostrado que la satisfacción de éstos sólo puede lograrse mediante la organización autónoma de las mujeres, y su movilización social y política) al no ser formulados directamente por las mujeres como los inherentes a la condición, permiten señalar que por efecto de la política pública se ha alcanzado un importante equilibrio de género entre las familias jornaleras, ya que 75\% de las variables medidas así lo demuestran.

Por último, llevar a cabo una diferenciación clara entre la condición y posición de las mujeres en un contexto determinado, permite tener una mejor noción de los efectos de las políticas públicas en materia de equidad de género. $\mathrm{Al}$ respecto, y de forma consecuente con lo planteado en los materiales y métodos, se indican los resultados obtenidos del proceso de construcción del subíndice ponderado de condición (SIPC), el subíndice 


\begin{tabular}{|c|c|c|c|c|c|}
\hline \multicolumn{6}{|c|}{$\begin{array}{c}\text { Cuadro } 6 \\
\text { Resultados de las variables de división del trabajo y control de } \\
\text { recursos por estrato }\end{array}$} \\
\hline \multirow[t]{2}{*}{ Variable } & \multirow{2}{*}{$\begin{array}{c}\text { Variable } \\
\text { operacionalizada }\end{array}$} & \multirow{2}{*}{ Indicador } & \multicolumn{3}{|c|}{ Lugar por estrato } \\
\hline & & & E1 & E2 & E3 \\
\hline \multirow{7}{*}{$\begin{array}{l}\text { División } \\
\text { del } \\
\text { trabajo }\end{array}$} & Tipo de actividad & En la localidad (encuesta) & 3 & 3 & 3 \\
\hline & & En campos agrícolas (encuesta) & 2 & 1 & 3 \\
\hline & Carga de trabajo & En localidad (encuesta) & 1 & 3 & 2 \\
\hline & & $\begin{array}{l}\text { En localidad (tiempos y } \\
\text { movimientos) }\end{array}$ & 1 & 2 & 3 \\
\hline & & En campos agrícolas (encuesta) & 3 & 1 & 2 \\
\hline & Ingreso y & En la localidad & 2 & 3 & 1 \\
\hline & $\begin{array}{l}\text { distribución } \\
\text { del gasto }\end{array}$ & En campos agrícolas & 3 & 2 & 1 \\
\hline \multirow[t]{2}{*}{ Subtotal } & & & 15 & 15 & 15 \\
\hline & $\begin{array}{l}\text { Administración } \\
\text { del gasto }\end{array}$ & $\%$ administración del gasto & 3 & 2 & 1 \\
\hline \multirow[t]{2}{*}{$\begin{array}{l}\text { Control de } \\
\text { recursos }\end{array}$} & $\begin{array}{l}\text { Acceso a instrum. } \\
\text { modernos }\end{array}$ & $\%$ acceso a menaje de casa & 3 & 1 & 2 \\
\hline & $\begin{array}{l}\text { Acceso a otros } \\
\text { bienes }\end{array}$ & $\begin{array}{l}\% \text { acceso a vivienda, tierra } \\
\text { e instrumentos de trabajo }\end{array}$ & 3 & 1 & 2 \\
\hline Subtotal & & & 9 & 4 & 5 \\
\hline
\end{tabular}

Fuente: Elaboración propia con base en datos de investigación de campo durante la primavera de 2009.

ponderado de posición (SIPP), y el índice ponderado de género (IPG) (cuadro 8).

De acuerdo con el análisis realizado y como se aprecia en el cuadro, la variable de educación en las necesidades prácticas, y las variables de control de recursos, acceso al poder y violencia familiar en las necesidades estratégicas, se muestra que $25 \%$ de las primeras y $75 \%$ de las segundas tienen un efecto positivo en la condición y posición de las mujeres jornaleras de la Sierra Negra.

\section{Conclusiones}

El esfuerzo de análisis y exposición en el capítulo precedente permite señalar, de manera categórica, que los proyectos para mujeres implemen- 


\section{Cuadro 7}

\section{Resultados de las variables de acceso al poder y violencia familiar por estrato}

\begin{tabular}{|c|c|c|c|c|c|}
\hline \multirow{2}{*}{ Variable } & \multirow{2}{*}{$\begin{array}{c}\text { Variable } \\
\text { operacionalizada }\end{array}$} & \multirow[t]{2}{*}{ Indicador } & \multicolumn{3}{|c|}{ Lugar por estrato } \\
\hline & & & E1 & E2 & E3 \\
\hline \multirow{3}{*}{$\begin{array}{l}\text { Acceso } \\
\text { al poder }\end{array}$} & \multirow{3}{*}{$\begin{array}{l}\text { Toma de } \\
\text { decisiones }\end{array}$} & Sobre actividades del hogar & 3 & 2 & 1 \\
\hline & & Sobre el ingreso & 3 & 2 & 1 \\
\hline & & $\begin{array}{l}\text { Participación en la vida pública } \\
\text { y gestión de apoyos } \\
\text { institucionales }\end{array}$ & 3 & 1 & 2 \\
\hline Subtotal & & & 9 & 5 & 4 \\
\hline $\begin{array}{l}\text { Violencia } \\
\text { familiar }\end{array}$ & $\begin{array}{l}\text { Violencia } \\
\text { psicológica y } \\
\text { física }\end{array}$ & $\begin{array}{l}\text { Prohibiciones, amenazas, } \\
\text { actitudes devaluatorias, celos, } \\
\text { intimidación, maltrato verbal, } \\
\text { físico y sexual }\end{array}$ & 3 & 2 & 1 \\
\hline Subtotal: & & & 3 & 2 & 1 \\
\hline
\end{tabular}

Fuente: Elaboración propia a partir de los resultados y discusión.

\section{Cuadro 8}

Resultados del SIPC, SIPP e IPG

\begin{tabular}{lcccr}
\hline \multicolumn{5}{c}{ Subindice ponderado de condición (SIPC) } \\
\hline \multicolumn{1}{c}{ Variable } & E1 & E2 & \multicolumn{1}{c}{ E3 } & \multicolumn{1}{c}{ Total } \\
\hline Alimentación & 0.165 & 0.45375 & 0.37125 & 0.99 \\
Educación & 0.37125 & 0.2475 & 0.12375 & 0.7425 \\
Salud & 0.20625 & 0.20625 & 0.0825 & 0.495 \\
Carencias materiales & 1.44375 & 1.485 & 0.9075 & 3.83625 \\
Subtotal & 0.02186 & 0.02392 & 0.01485 & 0.06063 \\
\hline & Subindice ponderado de posición (SIPP) & & \\
\hline Variable & E1 & E2 & E3 & Total \\
\hline División del trabajo & 0.61875 & 0.61875 & 0.61875 & 1.85625 \\
Control de recursos & 0.37125 & 0.165 & 0.20625 & 0.7425 \\
Acceso al poder & 0.37125 & 0.20625 & 0.165 & 0.7425 \\
Violencia familiar & 0.12375 & 0.0825 & 0.04125 & 0.2475 \\
Subtotal & 0.01485 & 0.01072 & 0.01031 & 0.03588 \\
Total (IPG) & 0.03671 & 0.03464 & 0.02516 & 0.09651 \\
\hline
\end{tabular}

Fuente: Elaboración propia con base en resultados y discusión. 
tados por la Sedesol-paja han contribuido a mejorar la condición o necesidades prácticas y la posición o necesidades estratégicas de género entre las mujeres jornaleras de la Sierra Negra.

Sin embargo, a partir de los análisis de los documentos relativos al programa y entrevistas a operadores del mismo, las formas de intervención de la Sedesol-paja en el estado de Puebla se muestran encaminadas a atender, a través de las mujeres, las necesidades de la familia y la comunidad más que de las propias mujeres, por lo que su enfoque es más instrumentalista que de género, queriendo dar respuesta o solución a los efectos de desigualdad, pero no así a las causas que la generan.

Para ilustrar lo anterior podemos señalar las mayores deficiencias en el balance calórico-proteico de las mujeres en comparación con los hombres del E1, ya que al hacerse cargo del trabajo extra que implican estos proyectos de apoyo por parte del programa, su actividad física o carga de trabajo se incrementa y, por ende, el tiempo para la recreación o el descanso disminuye drásticamente.

Otro ejemplo de ello es el balance de ingesta, que aunque se puede apreciar un incremento en el consumo de proteínas para el caso de las mujeres, se siguen conservando patrones -en menor medida- de distribución de alimentos priorizando al género masculino.

Tanto el balance positivo como el negativo entre el consumo y el gasto son inadecuados en el largo plazo para la salud humana; sin embargo, en lo que tiene que ver con el aspecto de inequidad de género, la escasez es un referente importante. En el único estrato donde se registra escasez o balance negativo entre consumo y gasto energético es en el E1, y dentro de éste el hombre es el que se encuentra menos afectado; esto es, aunque en menor grado, las mujeres mantienen un estatus de inequidad.

Si los proyectos instrumentados por la Sedesol se focalizaran a la posición y condición de la mujer, desde una perspectiva real de género, quizá los resultados serían distintos en términos de instrumentar políticas que incidan en las causas que provocan la inequidad entre hombres y mujeres, y no como un cambio indirecto que sí se observa en el mejoramiento de las necesidades prácticas y estratégicas, como es el caso de este tipo de proyectos focalizados al núcleo familiar.

\section{Bibliografía}

Bouserup, Ester (1970), Woman's role in economic development, George Allen and Unwin, Londres.

Carbajal, Carola (1998), “Desarrollo, ¿cómo?, ¿para quiénes?”, en María Antonieta Barrón Martínez (coord.), Manual metodológico de 
género. Libro I, Programa de Jornaleros Agrícolas de la Secretaría de Desarrollo Social-Fondo de Desarrollo de Naciones Unidas para la Mujer, México, pp. 12-15.

Conapo (Consejo Nacional de Población) (2006), Indices de marginalidad de los estados y municipios de la República, Conapo, México.

Coneval (Consejo Nacional de Evaluación de la Política de Desarrollo Social) (2007), Estimaciones con base en el segundo conteo de población y vivienda (2005), y la encuesta nacional de ingreso y gasto de los hogares (2005), Coneval, México.

Daltabuit, Magali (1988), Ecología humana en una comunidad de Morelos, Universidad Nacional Autónoma de México, México.

Daltabuit, Magali, Alicia Ríos y Fraterna Pérez (1988), Coba: estrategias adaptativas de tres familias mayas, Universidad Nacional Autónoma de México, México.

DOF (Diario Oficial de la Federación) (2007), "Reglas de operación del Programa de Atención a Jornaleros Agrícolas", DOF, México, pp. 2-9.

FAO-OMS (Organización Mundial para la Agricultura y la AlimentaciónOrganización Mundial de la Salud) (1985), Energy and protein requirements, FAO-OMS, Berna.

Hernández, Roberto, Carlos Fernández y Pilar Baptista (2000), Metodología de la investigación, McGraw Hill, México.

INEGI (Instituto Nacional de Estadística, Geografía e Informática) (2005), Anuario estadístico de los Estados Unidos Mexicanos, INEGI, México.

INEGI (Instituto Nacional de Estadística, Geografía e Informática) (2006), Anuario estadístico del estado de Puebla, INEGI-Gobierno del Estado de Puebla, Puebla.

McEachern, William (1998), Economía: una introducción contemporánea, Thomson, México.

Martínez, Beatriz, Silvia Martínez, Socorro Barrientos y Alberto Paredes (2002), Mujeres rurales y género: elementos para la transformación 
de su condición y posición, Colegio de Postgraduados, Puebla-Sedesol, México.

Molyneux, Maxine (1985), “¿Movilización sin emancipación? Intereses de la mujer, el Estado y la Revolución: el caso de Nicaragua”, en José Luis Coraggio y Carmen Diana Deere (coords.), La transformación difícil: la autodeterminación de los pequeños paises periféricos, Siglo XXI, México, pp. 227-254.

Moser, Caroline (1989), "La planificación de género en el Tercer Mundo: enfrentando las necesidades prácticas y estratégicas de género", en Virginia Guzmán, Patricia Portocarrero y Virginia Vargas (comps.), Una nueva lectura: género en el desarrollo, Centro de la Mujer Peruana Flora Tristán, Lima, pp. 37-54.

PNUD (Programa de Naciones Unidas para el Desarrollo) (2003), Informe sobre el desarrollo humano, PNUD-Tercer Mundo, Bogotá.

PNUD (Programa de Naciones Unidas para el Desarrollo) (2004), Informe sobre desarrollo humano en México, PNUD-Mundi-Prensa, México.

PR (Presidencia de la República) (2007), Estrategia: Vivir mejor, Gobierno de la República, México.

Pronjag-Puebla (1997), Diagnóstico de zonas intermedias, Coordinación Estatal del Programa de Atención a Jornaleros Agrícolas-Sedesol, México.

Sedesol (Secretaría de Desarrollo Social) (2007), Objetivos estratégicos de desarrollo social, Sedesol, México.

Villa-Issa, Manuel (2008), ¿Qué hacemos con el campo mexicano?, Colegio de Postgraduados-Colegio de Puebla, A.C.-Mundi Prensa, Puebla.

Viteri, Fernando (1971), "Determining energy costs of agricultural activities by respirometer and Energy Balance Techniques, Assessment of Daily Energy Expediture", en Robert B. Bradfield (ed.), Reprinted the American Journal of Clinical Nutrition of the American Society for Nutrition, vol. 14, American Society for Nutrition, Nueva York, pp. 418-430. 
Young, Kate (1997), "El potencial transformador en las necesidades prácticas: empoderamiento colectivo y el proceso de planificación”, en Magdalena León Gómez (comp.), Poder y empoderamiento de las mujeres, Tercer Mundo-Universidad Nacional de Colombia, Bogotá, pp. 105-107.

Recibido: 30 de enero de 2010. Reenviado: 24 de septiembre de 2010. Aceptado: 15 de febrero de 2011.

Humberto Piñón-Vargas. Es maestro en ciencias por el Colegio de Postgraduados, Campus Puebla, donde se graduó con mención honorífica. Realizó estudios de licenciatura en la Universidad Popular Autónoma del Estado de Puebla, donde se tituló como ingeniero agrónomo zootecnista, obteniendo reconocimiento como de los mejores estudiantes de México. Tiene experiencia laboral en programas de desarrollo del sector público federal: Sedesol, Diconsa y en la CDI; asimismo, ha tenido participación como consultor-investigador con el American Museum of Natural History, de Nueva York; en el Radiaton Center of Oregon State University, y en la ONG holandesa Bernard Van Leer. Actualmente es catedrático en la Universidad del Valle de México, Campus Puebla, y sus líneas de investigación son: género y desarrollo, migración infantil y jornaleros agrícolas. Entre sus publicaciones destacan: "El papel de los jornaleros agrícolas poblanos en el desarrollo rural", en Memorias del Congreso Nacional Agropecuario y Forestal, Universidad Autónoma Chapingo-Congreso Agrario Permanente, Chapingo, pp. 184-187 (1997); "Los niños jornaleros", en Avances y retrocesos: balance de una década. Quinto Informe sobre los derechos y situación de la infancia en México 19982000, Colectivo Mexicano de Apoyo a la Niñez, México, pp. 353-364 (1999); participó en el Primer Encuentro Nacional de Investigadores de Familia con la ponencia "Las familias jornaleras del ingenio de Atencingo", Tlaxcala, Tlax. (1997).

Esteban Martínez-Dajui. Es doctor en sociología por el Instituto de Filosofía y Ciencias Humanas de la Universidad Federal de Rio Grande do Sul en Porto Alegre, Brasil; realizó estudios de maestría en ciencias en estrategias para el desarrollo agrícola regional en el Colegio de Postgraduados. Es ingeniero agrónomo por el Instituto Tecnológico Agropecuario de Morelia, Michoacán. Actualmente es profesor-investigador asociado en el Colegio de Postgraduados, Campus Puebla, y desarrolla actividades de investigación relacionados con el desarrollo rural y micro- 
financiamiento. Entre sus publicaciones destacan: "Los grupos comunitarios de cajas de ahorro: una alternativa de crédito y ahorro para familias rurales pobres", en Estudios y propuestas para el medio rural, CP-UAIM, El Fuerte, Sinaloa, pp. 1-23 (2007); "El papel de las mujeres en la reproducción social en las unidades de producción familiar", en Estudios y propuestas para el medio rural, t. III, CP-UAIM, El Fuerte, Sinaloa, pp. 65-89 (2008); "El programa Prodesca-PSPs como medio para el desarrollo de capacidades organizativas en la producción hortícola", en Estudios y propuestas para el medio rural, t. IV, CP-UAIM, El Fuerte, Sinaloa, pp. 467-490 (2008); "Las cajas de ahorro comunitarias: un instrumento para la producción de alimentos de familias campesinas", en La seguridad alimentaria en Puebla: prioridad para el desarrollo, CP-SDR-Gobierno del Estado de Puebla, Puebla, pp. 189-197 (2008).

Miguel Sánchez-Hernández. Realizó estudios de ingeniero agrónomo en suelos en la Universidad Autónoma Chapingo; es maestro en divulgación agrícola por el Colegio de Postgraduados; es doctor por la Wisconsin State University, Madison, en el Programa de Desarrollo del Land Tenure. Tiene una carrera académica de investigación y servicio en el Colegio de Postgraduados de poco más de 30 años. Sus líneas de investigación están orientadas al entendimiento y estudio de los procesos organizativos campesinos y su relación con otros fenómenos también relevantes en el proceso de desarrollo rural; en ese sentido, es líder del grupo de investigación sobre organización campesina. Entre los productos más importantes, se pueden mencionar un buen número de tesis de maestría y doctorado que ha realizado con estudiantes de la especialidad en estrategias para el desarrollo agrícola regional, con quienes ha participado como consejero y/o asesor.

Gustavo Ernesto Barrientos-Lavín. Es licenciado en antropología física por la Escuela Nacional de Antropología; obtuvo el grado de maestro en la Universidad Nacional Autónoma de México el 26 de marzo de 1982, y el doctorado en la misma casa de estudios en octubre de 1995. Es investigador nacional, nivel I. Actualmente es profesor titular nivel III del Departamento de Antropología de la Universidad de las Américas, Puebla. Su línea de investigación actual es la disputa por el agua y el bosque en un municipio de Puebla. Entre sus publicaciones recientes destaca: en coautoría, Desarrollo de una metodología para la promoción de proyectos de extensión y vinculación universitaria con impacto económico y social, Secretaría de Economía-Universidad de las Américas, Puebla, México (2007). 